\title{
RIGHT ALTERNATIVE RINGS
}

\section{ERWIN RLEINFELD}

1. Introduction. A ring is defined to be right alternative in case $x y \cdot y-x \cdot y y=0$ is an identical relation in the ring. Right alternative algebras were first studied by A. A. Albert; who showed that $a$ semisimple, right alternative algebra over a field of characteristic 0 is alternative [1]. Another result concerns right alternative division rings, which are of geometrical interest since they arise as coordinate systems of certain projective planes in which a configuration weaker than Desargue's is assumed to hold. In this connection L. A. Skorniakov has shown that $a$ right alternative division ring of characteristic not 2 is alternative [2]. From the examples Albert presents in his paper it is apparent that one must abandon conventional approaches, as for instance the Peirce decomposition, in the study of a wider class of right alternative rings. It seems that a closer examination of the right alternative identity is thus called for.

In our treatment the associator plays a major role. We define the associator $(x, y, z)$ and commutator $(x, y)$ by $(x, y, z)=x y \cdot z-x \cdot y z$ and $(x, y)=x y-y x$. In this notation the right alternative identity becomes $(x, y, y)=0$, which in turn implies

$$
(x, y, z)=-(x, z, y) .
$$

Characteristic different from 2 will be tacitly assumed from now on.

Let $F$ be the free (non-associative) ring generated by $x_{1}, \cdots, x_{n}$. Suppose $R$ is any right alternative ring. We shall say the elements $t, u, v$ of $R$ form an alternative triple in case the following two conditions are satisfied: (i) There exist elements $\alpha\left[x_{1}, \cdots, x_{n}\right]$, $\gamma\left[x_{1}, \cdots, x_{n}\right], \delta\left[x_{1}, \cdots, x_{n}\right]$ in $F$ and elements $r_{1}, \cdots, r_{n}$ in $R$ such that $t=\alpha\left[r_{1}, \cdots, r_{n}\right], u=\gamma\left[r_{1}, \cdots, r_{n}\right], \quad v=\delta\left[r_{1}, \cdots, r_{n}\right]$; (ii) If $s_{1}, \cdots, s_{n}$ are any elements of an arbitrary alternative ring and $t^{\prime}=\alpha\left[s_{1}, \cdots, s_{n}\right], u^{\prime}=\gamma\left[s_{1}, \cdots, s_{n}\right], v^{\prime}=\delta\left[s_{1}, \cdots, s_{n}\right]$, then $\left(t^{\prime}, u^{\prime}, v^{\prime}\right)=0$. For our purposes $n$ may be taken equal to 2 . $R$ will be said to have property $(\mathrm{P})$ in case $t, u, v$ an alternative triple in $R$ and $(t, u, v)^{2}=0$ imply $(t, u, v)=0$.

It follows that an alternative ring automatically has property $(\mathrm{P})$. Our main result states that the converse also holds. In other words $a$ right alternative ring is alternative if and only if it has property (P). This theorem clearly generalizes Skorniakov's result.

For the sake of presenting a self-contained exposition, proofs of

Received by the editors March 17, 1953. 
Lemmas 1, 2, and 4 are given, even though these facts are essentially contained in Skorniakov's paper.

We use the symbols $a, b, c, w, x, y, z$ to denote arbitrary elements of $R$. In $\$ 3$ we assume that $R$ has property (P). It will be useful to know that two elements of an alternative ring generate an associative subring. ${ }^{1}$

2. Elementary properties. In order to facilitate the calculations we define the following functions:

$$
\begin{aligned}
f(w, x, y, z)= & (w x, y, z)-(w, x y, z)+(w, x, y z)-w(x, y, z) \\
& -(w, x, y) z, \\
g(x, w, y, z)= & (x, w, y z)+(x, y, w z)-(x, w, z) y-(x, y, z) w, \\
h(w, x, y, z)= & (w x, y, z)+(w, x,(y, z))-w(x, y, z)-(w, y, z) x .
\end{aligned}
$$

We shall show presently that all three functions are identically zero. In fact $f(w, x, y, z)=0$ holds in an arbitrary ring, as a direct consequence of the definition of the associator. By forming $f(x, y, y, z)$ $-f(x, z, y, y)+f(x, y, z, y)=0$ we obtain $2(x, y, y z)=2(x, y, z) y$. This implies

$$
(x, y, y z)=(x, y, z) y \text {. }
$$

In $f(x, z, y, y)=0$ we make use of (2), so that

$$
\left(x, z, y^{2}\right)=(x, y z+z y, y) \text {. }
$$

The identity $g(x, w, y, z)=0$ follows from a linearization of (2), obtained by replacing $y$ with $w+y$. Since $f(w, x, y, z)-g(w, z, x, y)$ $=h(w, x, y, z)$, it is clear that $h(w, x, y, z)=0$. We are now ready to prove a number of useful lemmas.

LEMMA 1. $(a b \cdot c) b=a(b c \cdot b)$.

Proof. We have $(a b \cdot c) b=(a, b, c) b+(a \cdot b c) b$. According to (2), $(a, b, c) b=(a, b, b c)=-(a, b c, b)=-(a \cdot b c) b+a(b c \cdot b)$. By comparing the two identities we prove the lemma.

Definition. For fixed elements $a, b$ in $R$ we define $S(a, b)$ as the set of all elements $x$ in $R$ which have the property $(x, a, b)=x(b, a)$.

It follows at once that $S(a, b)=S(b, a)$ and that $x$ belongs to $S(a, b)$ if and only if $x a \cdot b=x \cdot b a$. Moreover the elements of $S(a, b)$ form a group under addition.

${ }^{1}$ This is known in the literature as Artin's Theorem. For a proof see for instance R. H. Bruck and Erwin Kleinfeld, The structure of alternative division rings, Proc. Amer. Math. Soc. vol. 2 (1951) p. 888, or Zorn's original proof the latter refers to. 
LEMMA 2. If $x$ belongs to both $S(a, b)$ and $S(a, b a)$, then $x(a, a, b)=0$.

Proof. By Lemma $1,(x a \cdot b) a=x(a b \cdot a)$. Since $x$ belongs to $S(a, b)$ we have $(x a \cdot b) a=(x \cdot b a) a$, and because $x$ belongs to $S(a, b a),(x \cdot b a) a$ $=x(a \cdot b a)$. Comparison shows $x(a, b, a)=0$.

LEMmA 3. If both $y$ and $x y$ belong to $S(a, b)$, then $(x, a, b) y=0$.

Proof. By hypothesis we have the identities $(x y, a, b)=-x y$ $(a, b)$ and $x(y, a, b)=-x \cdot y(a, b)$. Substituting these in $h(x, y, a, b)$ $=0$ we obtain the lemma.

Lemma 4. For arbitrary $x,(x, a, b)$ and $(x, a, b)$ a are both contained in $S(a, b)$.

Proof. From (2) it follows that $\left(x, a, a b^{2}\right)=\left(x, a, b^{2}\right) a$, while from (3), $\left(x, a, b^{2}\right) a=(x, a b, b) a+(x, a, b) b \cdot a$. Through comparison of these two identities with $g(x, a, a b, b)=0$ we are led to $(x, a, b) b \cdot a$ $=(x, a, b) \cdot a b$, which shows that $(x, a, b)$ belongs to $S(a, b)$.

From (2) we also obtained the identities $\left(x, b a, b a^{2}\right)=(x, b a, a) \cdot b a$ and $\left(x, b, b a^{3}\right)=\left(x, b, a^{8}\right) b$. These together with $g\left(x, b a, b, a^{2}\right)=0$ imply that $\left(x, b, a^{3}\right) b-\left(x, b a, a^{2}\right) b=(x, a b, a) b a$. Comparison of this last identity with $g\left(x, b, a^{2}, a\right) b=0$ proves that $(x, a b, a) \cdot b a$ $=(x, b, a) a^{2} \cdot b=(x, a b, a) a \cdot b$, which is precisely what is needed to assert that $(x, a, b) a$ belongs to $S(a, b)$. This completes the proof.

3. Main section. Henceforth we need to assume that our ring $R$ has property $(\mathrm{P})$. We adopt the notation $p=(a, a, b), q=(a, b)$. Our aim is to produce situations in which property $(P)$ can be applied. Eventually we must establish $p=0$. The next three lemmas provide some of the essential tools toward this goal.

LEMMA 5. (i) $(q, a, b)=0$, (ii) $((a, b), a, c)=-((a, c), a, b)$, (iii) $(x a \cdot b-x \cdot b a, a, b)=0$, (iv) $\left(p^{2}, a, q\right)=0=\left(p^{2}, a, a b\right)=\left(p^{2}, a, b a\right)$.

Proof. From Lemma 4 we deduce that $-(q, a, b) q=((q, a, b), a, b)$, so that $(q, a, b) q$ is contained in $S(a, b)$. Since $\left(q^{2}, a, b\right)$ is also contained in $S(a, b)$ we use $h(q, q, a, b)=\left(q^{2}, a, b\right)-q(q, a, b)-(q, a, b) q=0$ to prove that $q(q, a, b)$ is a member of $S(a, b)$. We can now apply Lemma 3 to obtain $(q, a, b)^{2}=0$. Property (P) may now be applied in the obvious fashion and (i) follows. Part (ii) is just a linearization of (i), obtained by replacing $b$ with $b+c$. Now $h(x, q, a, b)=0$ together with (i) implies that $(x q, a, b)=(x, a, b) q$, while, by Lemma $4,(x, a, b) q$ $=-((x, a, b), a, b)$. This suffices for the proof of (iii). As a consequence of Lemma $4,(p a, a, b)=-p a \cdot q$ and $(p, a, b) a=-p q \cdot a \cdot$. Comparison with $h(p, a, a, b)=0$ proves that $p q \cdot a-p \cdot a q=p^{2}$. It is clear 
that $p^{2}$ may also be expressed as $p^{2}=-p(a, b, a)=-p(a b \cdot a)$ $+p(a \cdot b a)$. Since Lemma 1 implies that $p(a b \cdot a)=(p a \cdot b) a$, and, by Lemma 4, $p a \cdot b=p \cdot b a$, we obtain $p^{2}=-(p \cdot b a) a+p(a \cdot b a)$. Both of these expressions of $p^{2}$, when combined with (iii), imply (iv). This completes the proof of the lemma.

LemMA 6. If $a b=0$, then $(b, a, b)=b a \cdot b=0$ and $(b a)^{2}=0$.

Proof. Let $s=(b, a, b)$. Then $0=s \cdot a b=s b \cdot a$. Consequently 0 $=(s b \cdot a) b=s(b a \cdot b)$, using Lemma 1 . Since $s(b \cdot a b)=0$ we obtain $s^{2}=0$, so that $s=0$. Also $0=((a, b), a, b)=-(b a, a, b)=(b a, b, a)=(b a \cdot b) a$ $-(b a)^{2}$, with the aid of Lemma 5 (i). However it follows from Lemma 1 that $(b a \cdot b) a=b(a b \cdot a)=0$. Therefore $(b a)^{2}=0$.

$$
\begin{gathered}
\text { LEMMA 7. (i) } 2(q, a, q)=q p-p q, \quad(\mathrm{ii}) \quad(q p-p q) p=0 \\
{[p(p q+q p)] p=0 \text {. }}
\end{gathered}
$$

Proof. With the use of Lemma 5, part (i) and (ii), we can prove that $r=(q, a, q)=-(q, a, b a)=((a, b a), a, b)$. Thus $r$ belongs to both $S(a, b)$ and $S(a, b a)$, hence by Lemma 2, $r p=0$. From this we see that (i) implies (ii). Since $h(q, a, a, b)=0$ we have $(q a, a, b)+r$ $=q p$, while it follows from $h(a, q, a, b)=0$ that $(a q, a, b)=p q$. Through Lemma 5 (ii) we see that $((a, q), a, b)=-r$. Combining the last three identities one deduces that $2 r=q p-p q$, as was to be shown. Only part (iii) remains to be proven. From $h(p, p, a, b)=0$ we obtain $\left(p^{2}, a, b\right)$ $+(p, p, q)=p(p, a, b)+(p, a, b) p$. Now $(p, a, b)=-p q$ by Lemma 4, so that $\left(p^{2}, a, b\right)=(p, q, p)-p \cdot p q-p q \cdot p=-p(p q+q p)$. It will suffice to show that $\left(p^{2}, a, b\right) p=0$. However combining $h\left(p^{2}, a, a, b\right)=0$ with Lemma 5 (iv) we conclude that $\left(p^{2} a, a, b\right)=p^{8}$, so that $p^{8}$ is in $S(a, b)$. At this point Lemma 3 can be applied to finish the proof.

From this point on we proceed directly with the proof of the main result. From Lemma 7 (iii) we know that $[p(p q+q p)] p=0$. Through a combination of Lemma 6 and Lemma 7 (ii) we obtain that $[p(p q-q p)] p=0$. We conclude that

$$
(p \cdot p q) p=0=(p \cdot q p) p .
$$

But then $(p, p q, p)=-p(p q \cdot p)=-p(q p \cdot p)$ because of Lemma 7 (ii). On the other hand $-p(q p \cdot p)=(p, q p, p)$, so that $(p, p,(p, q))=0$. Since $h(p, p, p, q)=0$ we can write $\left(p^{2}, p, q\right)=p(p, p, q)+(p, p, q) p$. Clearly, by Lemma $4,\left(p^{2}, p, q\right)$ and $(p, p, q) p$ belong to $S(p, q)$. This, in combination with Lemma 3 , implies $(p, p, q)^{2}=0$, thus

$$
(p, p, q)=0
$$

From (4), (5), and Lemma 7 (ii) we can obtain 


$$
p^{2} q p=p q p^{2}=q p^{3}=0,
$$

and in fact it is immaterial which way the products in (6) are associated. Using $h\left(p^{2}, a, a, b\right)=0$ and Lemma 5 (iv) we deduce that $\delta\left(p^{2}, a, a, b\right)=p^{3}$. Therefore $h\left(p^{2} a, q, a, b\right)=0$ implies that $\left(p^{2} a \cdot q, a, b\right)$ $=\left(p^{2} a, a, b\right) q=p^{3} q$. Inasmuch as $q p^{8}=0$ from (6), Lemma 6 implies $\left(p^{3} q\right)^{2}=0$. Since $p^{3} q$ is an associator of the above type we may use property (P) and obtain $p^{8} q=0$. Because of (5) we can show that $p^{4} q=0$. Hence $p^{2}\left(p^{2}, a, b\right)=p^{2}(-p p q-p q p)=0$, using $h(p, p, a, b)$ $=0$. This allows us to utilize Lemma 3 and we infer that $\left(p^{2}, a, b\right)^{2}=0$, so that

$$
\left(p^{2}, a, b\right)=0 .
$$

From Lemma 7 (i) we get the identity $q p=2(q, a, q)+p q$ $=-2((a, q), a, b)-(p, a, b)=(-2(a, q)-p, a, b)$. Thereby $q p+p q$ $=(-2(a, q)-2 p, a, b)$ and consequently $p q+q p$ is contained in $S(a, b)$. Now from $h(p, p, a, b)=0,(7)$, and (5) it follows that 0 $=p(p, a, b)+(p, a, b) p=-p(p q+q p)$, so that

$$
p(p q+q p)=0 \text {. }
$$

Combining (8) with Lemma 3 we obtain $(p, a, b)(p q+q p)=0$ and hence

$$
p q(p q+q p)=0 \text {. }
$$

Also $(q p)(p q+q p)=(q, p, q p+p q)=\left(q, p^{2}, q\right)$, employing (8) and (3). Since $h\left(q, p^{2}, a, b\right)=0$, we have $\left(q p^{2}, a, b\right)+\left(q, p^{2}, q\right)=0$. Now through Lemma 7 (ii) and (8) it can be seen that

$$
q p^{2}=p q p=-p^{2} q,
$$

and thus $\left(q, p^{2}, q\right)=-\left(q p^{2}, a, b\right)=\left(p^{2} q, a, b\right)$. Moreover from $h\left(p^{2}, q, a, b\right)=0$, Lemma 5 (i), and (7) it can be proven that $\left(p^{2} q, a, b\right)$ $=0$. Therefore $(q p)(p q+q p)=0$. In conjunction with (9) this means $(p q+q p)^{2}=0$. Once again Property $(\mathrm{P})$ may be invoked and we obtain

$$
p q+q p=0 \text {. }
$$

From (11) we are led to $q p^{2}=p^{2} q$, which together with (10) implies that $p^{2} q=p q p=q p^{2}=0$. However $p q$ is a member of $S(a, b)$ and so is $p^{2} q$. Again Lemma 3 can be used in order to prove that $(p, a, b) \cdot p q$ $=-(p q)^{2}=0$, so that $p q=0$. Hence with (11) and Lemma 4 we have

$$
(p, a, b)=0=p q=q p .
$$

Since $(p, a, b)=0$, we can linearize and obtain $((a, a, c), a, b)$ 
$=-((a, a, b), a, c)$. In particular $(p a, a, b)=((a, a, a b), a, b)$ $=-(p, a, a b)=-(p, a, b) a=0$. From $h(p, a, a, b)=0$ it now follows that $(p, a, q)=p^{2}$. Since $(p, a, a b)=(p, a, b) a=0$ it must be that $p^{2}$ $=-(p, a, b a)$ and hence $p^{2}$ is contained in $S(a, b a)$. Also $p^{2} q=0$ and $\left(p^{2}, a, b\right)=0$, from (12) and (7) respectively, so that $p^{2}$ is a member of $S(a, b)$ as well. Then by Lemma $2, p^{3}=0$. Thereby $p^{4}=0$, so that $p^{2}=0$ and then $p=0$, using property $(\mathrm{P})$ twice in a row. We may.now state:

THEOREM. $A$ right alternative ring of characteristic not 2 is alternative if and only if it has property (P).

If one desires to assume a stronger hypothesis than property $(\mathrm{P})$, then our proof may be considerably shortened. Suppose we assume that $R$ has no nilpotent elements. In conjunction with Lemma 6 we can then readily show that $a b \cdot a=0$ implies $a b=b a=0$. Therefore (12) follows directly from Lemma 7. In case $R$ has no divisors of zero even greater economy can be achieved.

Our main result has one drawback. Being dependent on calculations which involve associators, the final result involves associators and this does not seem to connect in an obvious way with the usual structure theory of rings. There is some hope however that both theories have a common denominator, which will lead to further results on the subject.

\section{BiBLIOGRAPHY}

1. A. A. Albert, On the right alternative algebras, Ann. of Math. vol. 50 (1949) pp. 318-328.

2. L. A. Skorniakov, Right alternative fields, Izvestia Akad. Nauk SSSR Ser. Mat. vol. 15 (1951) pp. 177-184.

University of Chicago 